\title{
Recent Results on the Cauchy Problem for Focusing and Defocusing Gross-Pitaevskii Hierarchies
}

\author{
Thomas Chen * and Nataša Pavlović \\ University of Texas at Austin, Department of Mathematics, Austin, TX 78712, USA
}

\begin{abstract}
In this paper, we review some of our recent results in the study of the dynamics of interacting Bose gases in the Gross-Pitaevskii (GP) limit. Our investigations focus on the wellposedness of the associated Cauchy problem for the infinite particle system described by the GP hierarchy.
\end{abstract}

Key words: Bose gas, Gross-Pitaevskii limit, BBGKY hierarchy, nonlinear Schrödinger equations, mean field limit

AMS subject classification: $35 \mathrm{Q} 55,81 \mathrm{~V} 70$

\section{Introduction}

The mathematical analysis of interacting Bose gases has experienced enormous progress in recent years. One of the motivating and most fundamental questions in this field addresses the rigorous proof of Bose-Einstein condensation; for some recent landmark results in this area, we refer to the works of Lieb, Seiringer, Yngvason, and their collaborators which have initiated much of the current interest in the field, see [2, 23, 24, 25] and the references therein.

Another main direction of research focuses on the effective mean field dynamics of interacting Bose gases. Due to the extremely large number of degrees of freedom, the dynamics of such systems is exceedingly complicated, and difficult to access mathematically. However, it is possible to extract very important information about its dynamics on the average, by determining appropriate mean field limits. Those are typically described by solutions of the nonlinear Schrödinger (NLS) or nonlinear Hartree (NLH) equation. In recent years, there has been remarkable progress in the

*Corresponding author. E-mail: tc@ math.utexas.edu 
mathematically rigorous derivation of mean field limits of interacting Bose gases. For some fundamental results in this field, we refer to the works of Erdös, Schlein and Yau in $[12,13,14]$ which have initiated much of the current interest in this area. See also $[21,22,26]$ and the references therein; see also $[1,3,11,15,16,17,18,19,20,28]$.

The strategy of $[12,13,14]$ for derivation of cubic NLS involves the following main steps, which are presented in more detail in Section 2.:

(i) Based on the Schrödinger evolution of the given $N$-body system of bosons, one derives the associated BBGKY hierarchy of marginal density matrices.

(ii) Then one takes the limit $N \rightarrow \infty$, whereupon the BBGKY hierarchy tends to an infinite hierarchy of marginal density matrices referred to as the Gross-Pitaevskii (GP) hierarchy.

(iii) Finally, one proves the uniqueness of solutions for the GP hierarchy, which implies that for factorized initial data, the solutions of the GP hierarchy are factorized with individual factors satisfying the NLS.

Inspired by the fascinating progress that has been achieved on the derivation of the cubic NLS, we got interested in the topic and started to explore certain aspects of it. In this paper we give a short summary of our recent results.

In particular, we start by giving a review of the derivation of the cubic NLS which is presented in Section 2.. In that context, in Section 3., we present a review of our recent derivation of the quintic NLS. In Section 4. we revisit the GP hierarchy and describe the new way to look at the hierarchy which is based on introducing a topology that allows one to apply a fixed point argument and establish local existence and uniqueness of solutions. Furthermore, in Section 4. we give a brief summary of our recent results addressing finite time blow-up of solutions to the GP hierarchy as well as global well-posedness.

\section{Derivation of the cubic NLS}

We outline below the main steps along which the defocusing cubic NLS is derived as the mean field limit for a gas of bosons with repelling pair interactions, following [12, 13, 14]. It is currently not known how to obtain analogous results for the case of attractive interactions.

\subsection{From $N$-body Schrödinger to BBGKY}

Let $\psi_{N} \in L^{2}\left(\mathbb{R}^{d N}\right)$ denote the wave function describing $N$ bosons in $\mathbb{R}^{d}$. To comply with BoseEinstein statistics, $\psi_{N}$ is invariant with respect to permutations $\pi \in S_{N}$, which act by interchanging the particle variables,

$$
\psi_{N}\left(x_{\pi(1)}, x_{\pi(2)}, \ldots, x_{\pi(N)}\right)=\psi_{N}\left(x_{1}, x_{2}, \ldots, x_{N}\right) .
$$

We denote $L_{s}^{2}\left(\mathbb{R}^{d N}\right):=\left\{\psi_{N} \in L^{2}\left(\mathbb{R}^{d N}\right) \mid \psi_{N}\right.$ satisfies (2.1) $\}$. 
The dynamics of the system is determined by the Schrödinger equation

$$
i \partial_{t} \psi_{N}=H_{N} \psi_{N}
$$

where the Hamiltonian $H_{N}$ is assumed to be a self-adjoint operator acting on the Hilbert space $L_{s}^{2}\left(\mathbb{R}^{d N}\right)$, of the form

$$
H_{N}=\sum_{j=1}^{N}\left(-\Delta_{x_{j}}\right)+\frac{1}{N} \sum_{1 \leq i<j \leq N} V_{N}\left(x_{i}-x_{j}\right),
$$

with $V_{N}(x)=N^{d \beta} V\left(N^{\beta} x\right)$ and $V \in W^{r, s}\left(\mathbb{R}^{d}\right)$ spherically symmetric, for some suitable $r$, $s$, and for $\beta \in(0,1)$ sufficiently small.

The solution of the Schrödinger equation

$$
i \partial_{t} \Psi_{N, t}=H_{N} \Psi_{N, t}
$$

with initial condition $\Psi_{N} \in L_{s}^{2}\left(\mathbb{R}^{d N}\right)$, determines the $N$-particle density matrix

$$
\gamma_{N}\left(t ; \underline{x}_{N} ; \underline{x}_{N}^{\prime}\right)=\overline{\Psi_{N, t}\left(\underline{x}_{N}\right)} \Psi_{N, t}\left(\underline{x}_{N}^{\prime}\right)
$$

and its associated $k$-particle marginal density matrices

$$
\gamma_{N, t}^{(k)}\left(t ; \underline{x}_{k} ; \underline{x}_{k}^{\prime}\right)=\int d \underline{x}_{N-k} \gamma_{N}\left(t ; \underline{x}_{k}, \underline{x}_{N-k} ; \underline{x}_{k}^{\prime}, \underline{x}_{N-k}\right),
$$

for $k=1, \ldots, N$, where $\underline{x}_{k}=\left(x_{1}, \ldots, x_{k}\right), \underline{x}_{N-k}=\left(x_{k+1}, \ldots, x_{N}\right)$.

The time evolution of the density matrix $\gamma_{N}$ is determined by the Heisenberg equation

$$
i \partial_{t} \gamma_{N}(t)=\left[H_{N}, \gamma_{N}(t)\right]
$$

which is equivalent to

$$
\begin{aligned}
i \partial_{t} \gamma_{N}\left(t, \underline{x}_{N}, \underline{x}_{N}^{\prime}\right)= & -\left(\Delta_{\underline{x}_{N}}-\Delta_{\underline{x}_{N}^{\prime}}\right) \gamma_{N}\left(t, \underline{x}_{N}, \underline{x}_{N}^{\prime}\right) \\
& +\frac{1}{N} \sum_{1 \leq i<j \leq N}\left[V_{N}\left(x_{i}-x_{j}\right)-V_{N}\left(x_{i}^{\prime}-x_{j}^{\prime}\right)\right] \gamma_{N}\left(t, \underline{x}_{N}, \underline{x}_{N}^{\prime}\right),
\end{aligned}
$$

expressed in terms of the associated integral kernel. Accordingly, the $k$-particle marginals satisfy the BBGKY hierarchy

$$
\begin{aligned}
& i \partial_{t} \gamma^{(k)}\left(t, \underline{x}_{k} ; \underline{x}_{k}^{\prime}\right)=-\left(\Delta_{\underline{x}_{k}}-\Delta_{\underline{x}_{k}^{\prime}}\right) \gamma^{(k)}\left(t, \underline{x}_{k}, \underline{x}_{k}^{\prime}\right) \\
&+\frac{1}{N} \sum_{1 \leq i<j \leq k}\left[V_{N}\left(x_{i}-x_{j}\right)-V_{N}\left(x_{i}^{\prime}-x_{j}^{\prime}\right)\right] \gamma^{(k)}\left(t, \underline{x}_{k} ; \underline{x}_{k}^{\prime}\right) \\
&+\frac{N-k}{N} \sum_{i=1}^{k} \int d x_{k+1}\left[V_{N}\left(x_{i}-x_{k+1}\right)-V_{N}\left(x_{i}^{\prime}-x_{k+1}\right)\right] \\
& \gamma^{(k+1)}\left(t, \underline{x}_{k}, x_{k+1} ; \underline{x}_{k}, x_{k+1}^{\prime}\right)
\end{aligned}
$$

where $\Delta_{\underline{x}_{k}}:=\sum_{j=1}^{k} \Delta_{x_{j}}$, and similarly for $\Delta_{\underline{x}_{k}^{\prime}}$. We note that the size of the sum weighted by combinatorial factor in (2.9) is $\approx \frac{k^{2}}{N} \rightarrow 0$, and in (2.10) is $\frac{k(N-k)}{N} \rightarrow k$ as $N \rightarrow \infty$. Accordingly, it can be shown that, for fixed $k,(2.9)$ disappears in the limit $N \rightarrow \infty$ described below, while (2.10) survives. 


\subsection{From BBGKY to GP}

It is proven in $[12,13,14]$ that, for a suitable topology on the space of marginal density matrices, and as $N \rightarrow \infty$, one can extract convergent subsequences $\gamma_{N}^{(k)} \rightarrow \gamma^{(k)}$ for $k \in \mathbb{N}$, which satisfy the infinite limiting hierarchy

$$
\begin{aligned}
i \partial_{t} \gamma^{(k)}\left(t, \underline{x}_{k} ; \underline{x}_{k}^{\prime}\right)= & -\left(\Delta_{\underline{x}_{k}}-\Delta_{\underline{x}_{k}^{\prime}}\right) \gamma^{(k)}\left(t, \underline{x}_{k} ; \underline{x}_{k}^{\prime}\right) \\
& +b_{0} \sum_{j=1}^{k} B_{j, k+1} \gamma^{k+1}\left(t, \underline{x}_{k} ; \underline{x}_{k}^{\prime}\right),
\end{aligned}
$$

which is referred to as the Gross-Pitaevskii (GP) hierarchy. Here,

$$
\begin{aligned}
& \left(B_{j, k+1} \gamma^{k+1}\right)\left(t, \underline{x}_{k} ; \underline{x}_{k}^{\prime}\right) \\
& :=\int d x_{k+1} d x_{k+1}^{\prime}\left[\delta\left(x_{j}-x_{k+1}\right) \delta\left(x_{j}-x_{k+1}^{\prime}\right)-\delta\left(x_{j}^{\prime}-x_{k+1}\right) \delta\left(x_{j}^{\prime}-x_{k+1}^{\prime}\right)\right] \\
& \gamma^{(k+1)}\left(t, \underline{x}_{k}, x_{k+1} ; \underline{x}_{k}^{\prime}, x_{k+1}^{\prime}\right),
\end{aligned}
$$

and $b_{0}=\int V(x) d x$. The interaction term here is obtained from the limit of (2.10) as $N \rightarrow \infty$, using that $V_{N}(x) \rightarrow b_{0} \delta(x)$ weakly. We will set $b_{0}=1$ in the sequel.

\subsection{NLS and factorized solutions of GP}

The link between the original bosonic $N$-body system and solutions of the NLS is established as follows. Given factorized $k$-particle marginals

$$
\gamma_{0}^{(k)}\left(\underline{x}_{k} ; \underline{x}_{k}^{\prime}\right)=\left|\phi_{0}\right\rangle\left\langle\left.\phi_{0}\right|^{\otimes k}:=\prod_{j=1}^{k} \phi_{0}\left(x_{j}\right) \overline{\phi_{0}\left(x_{j}^{\prime}\right.}\right)
$$

at initial time $t=0$, with $\phi_{0} \in H^{1}\left(\mathbb{R}^{d}\right)$, one can easily verify that the solution of the GP hierarchy remains factorized for all $t \in I \subseteq \mathbb{R}$,

$$
\gamma^{(k)}\left(t, \underline{x}_{k} ; \underline{x}_{k}^{\prime}\right)=\prod_{j=1}^{k} \phi\left(t, x_{j}\right) \overline{\phi\left(t, x_{j}^{\prime}\right)},
$$

if $\phi(t) \in H^{1}\left(\mathbb{R}^{d}\right)$ solves the defocusing cubic NLS,

$$
i \partial_{t} \phi=-\Delta_{x} \phi+|\phi|^{2} \phi,
$$

for $t \in I$, and $\phi(0)=\phi_{0} \in H^{1}\left(\mathbb{R}^{d}\right)$.

Solutions of the GP hierarchy are studied in spaces of $k$-particle marginals with norms $\left\|\gamma^{(k)}\right\|_{H_{k}^{1}}^{\sharp}:=$ $\operatorname{Tr}\left(S^{(k)} \gamma^{(k)}\right)<\infty$ or $\left\|\gamma^{(k)}\right\|_{H_{k}^{1}}:=\left(\operatorname{Tr}\left(S^{(k)} \gamma^{(k)}\right)^{2}\right)^{1 / 2}<\infty$ where $S^{(k)}:=\prod_{j=1}^{k}\left\langle\nabla_{x_{j}}\right\rangle\left\langle\nabla_{x_{j}^{\prime}}\right\rangle$, and 
$H_{k}^{\alpha} \equiv H^{\alpha}\left(\mathbb{R}^{d k} \times \mathbb{R}^{d k}\right)$ for brevity. While the existence of factorized solutions can be easily obtained, as outlined above, the question remains whether solutions of the GP hierarchy are also unique.

The proof of uniqueness of solutions of the GP hierarchy is the most difficult part in the program outlined above, and it was originally accomplished by Erdös, Schlein and Yau in [12, 13, 14] by use of sophisticated Feynman graph expansion methods. In [21] Klainerman and Machedon proposed an alternative method, inspired by $[12,13,14]$, for proving uniqueness in $d=3$, based on use of space-time bounds on the density matrices and introduction of an elegant "board game" argument whose purpose is to organize the relevant combinatorics related to expressing solutions of the GP hierarchy using iterated Duhamel formulas. For the approach developed in [21], the authors make the assumption of a particular a priori spacetime bound on the density matrices. In the work [22] of Kirkpatrick, Schlein, and Staffilani, the corresponding problem in $d=2$ is solved, and the assumption made in [21] is replaced by a spatial a priori bound which is proven in [22].

\section{Derivation of the quintic NLS}

Motivated by the work on the derivation of the cubic NLS described above, we derive the quintic NLS as the mean field limit of the manybody quantum dynamics of interacting Boson gases in [6].

More precisely, we consider a system of $N$ bosons whose dynamics is generated by the Hamiltonian

$$
H_{N}:=\sum_{j=1}^{N}\left(-\Delta_{x_{j}}\right)+\frac{1}{N^{2}} \sum_{1 \leq i<j<k \leq N} N^{2 d \beta} V\left(N^{\beta}\left(x_{i}-x_{j}\right), N^{\beta}\left(x_{i}-x_{k}\right)\right),
$$

on the Hilbert space $L_{s}^{2}\left(\mathbb{R}^{d N}\right)$. We assume that the translation-invariant three-body potential $V$ has the properties

$$
V \geq 0 \quad, \quad V(x, y)=V(y, x) \quad, \quad V \in W^{3, p}\left(\mathbb{R}^{2 d}\right)
$$

for $2 d<p \leq \infty$.

We note that since

$$
\begin{aligned}
U\left(x_{1}-x_{2}, x_{2}-x_{3}, x_{1}-x_{3}\right) & =U\left(x_{1}-x_{2},-\left(x_{1}-x_{2}\right)+\left(x_{1}-x_{3}\right), x_{1}-x_{3}\right) \\
& \equiv V\left(x_{1}-x_{2}, x_{1}-x_{3}\right)
\end{aligned}
$$

every translation invariant three-body interaction potential $U$ can be written as (3.1).

As in the case described in Section 2., the solution of the Schrödinger equation

$$
i \partial_{t} \Psi_{N, t}=H_{N} \Psi_{N, t}
$$

with initial condition $\Psi_{N} \in L_{s}^{2}\left(\mathbb{R}^{d N}\right)$ determines the $N$-particle density matrix

$$
\gamma_{N}\left(t ; \underline{x}_{N} ; \underline{x}_{N}^{\prime}\right)=\overline{\Psi_{N, t}\left(\underline{x}_{N}\right)} \Psi_{N, t}\left(\underline{x}_{N}^{\prime}\right),
$$


and its associated $k$-particle marginals

$$
\gamma_{N, t}^{(k)}\left(t ; \underline{x}_{k} ; \underline{x}_{k}^{\prime}\right)=\int d \underline{x}_{N-k} \gamma_{N}\left(t ; \underline{x}_{k}, \underline{x}_{N-k} ; \underline{x}_{k}^{\prime}, \underline{x}_{N-k}\right),
$$

for $k=1, \ldots, N$. Then, the corresponding BBGKY hierarchy is given by

$$
\begin{aligned}
& i \partial_{t} \gamma_{N, t}^{(k)}=\sum_{j=1}^{k}\left[-\Delta_{x_{j}}, \gamma_{N, t}^{(k)}\right]+\frac{1}{N^{2}} \sum_{1 \leq i<j<\ell \leq k}\left[V_{N}\left(x_{i}-x_{j}, x_{i}-x_{\ell}\right), \gamma_{N, t}^{(k)}\right] \\
& +\frac{(N-k)}{N^{2}} \sum_{1 \leq i<j \leq k} \operatorname{Tr}_{k+1}\left[V_{N}\left(x_{i}-x_{j}, x_{i}-x_{k+1}\right), \gamma_{N, t}^{(k+1)}\right] \\
& +\frac{(N-k)(N-k-1)}{N^{2}} \sum_{j=1}^{k} \operatorname{Tr}_{k+1} \operatorname{Tr}_{k+2}\left[V_{N}\left(x_{j}-x_{k+1}, x_{j}-x_{k+2}\right), \gamma_{N, t}^{(k+2)}\right]
\end{aligned}
$$

where $V_{N}(x, y):=N^{2 d \beta} V\left(N^{\beta} x, N^{\beta} y\right)$.

In the limit $N \rightarrow \infty$, the sums weighted by combinatorial factors have the following size. In the first interaction term on the rhs of (3.2), $\frac{k^{3}}{N^{2}} \rightarrow 0$ for every fixed $k$ and sufficiently small $\beta$. For the second term $\frac{(N-k) k^{2}}{N^{2}} \rightarrow 0$. For the third interaction term, $\frac{(N-k)(N-k-1)}{N^{2}} \rightarrow 1$ for every fixed $k$. Accordingly, a rigorous argument shows that in the limit $N \rightarrow \infty$, one obtains the infinite GP hierarchy

$$
i \partial_{t} \gamma_{\infty, t}^{(k)}=\sum_{j=1}^{k}\left[-\Delta_{x_{j}}, \gamma_{\infty, t}^{(k)}\right]+b_{0} \sum_{j=1}^{k} B_{j ; k+1, k+2} \gamma_{\infty, t}^{(k+2)}
$$

for the coupling constant $b_{0}=\int d x_{1} d x_{2} V\left(x_{1}, x_{2}\right)$. The "contraction operator" is given by

$$
\begin{aligned}
B_{j ; k+1, k+2} \gamma_{\infty, t}^{(k+2)}\left(x_{1}, \ldots, x_{k} ; x_{1}^{\prime}, \ldots, x_{k}^{\prime}\right) & \\
:= & \int d x_{k+1} d x_{k+1}^{\prime} d x_{k+2} d x_{k+2}^{\prime} \\
& {\left[\delta\left(x_{j}-x_{k+1}\right) \delta\left(x_{j}-x_{k+1}^{\prime}\right) \delta\left(x_{j}-x_{k+2}\right) \delta\left(x_{j}-x_{k+2}^{\prime}\right)\right.} \\
\quad & \left.\delta\left(x_{j}^{\prime}-x_{k+1}\right) \delta\left(x_{j}^{\prime}-x_{k+1}^{\prime}\right) \delta\left(x_{j}^{\prime}-x_{k+2}\right) \delta\left(x_{j}^{\prime}-x_{k+2}^{\prime}\right)\right] \\
& \gamma_{\infty, t}^{(k+2)}\left(x_{1}, \ldots, x_{k}, x_{k+1}, x_{k+2} ; x_{1}^{\prime}, \ldots, x_{k}^{\prime}, x_{k+1}^{\prime}, x_{k+2}^{\prime}\right) .
\end{aligned}
$$

It is easy to see that a factorized state

$$
\gamma_{\infty, t}^{(k)}=\left|\phi_{t}\right\rangle\left\langle\left.\phi_{t}\right|^{\otimes k}\right.
$$

is a solution of (3.3) if and only if $\phi_{t}$ satisfies the quintic NLS

$$
i \partial_{t} \phi_{t}+\Delta_{x} \phi_{t}-b_{0}\left|\phi_{t}\right|^{4} \phi_{t}=0
$$


with $\phi_{0} \in L^{2}\left(\mathbb{R}^{d}\right)$.

In [6] we prove the convergence of the BBGKY hierarchy to the GP hierarchy by adapting the arguments from the work of Erdös-Schlein-Yau [12, 13, 14]. In order to prove the uniqueness of the limiting hierarchy, we expand the approach introduced by Klainerman-Machedon [21] and subsequently used by Kirkpatrick-Schlein-Staffilani [22]. Thereby, we obtain a mathematically rigorous derivation of the quintic NLS.

We remark that three-body interactions can for instance emerge in situations where the Bose gas interacts with a background field of radiation (photons) or lattice excitations (phonons). Averaging over the latter will typically lead to a linear combination of effective $n$-particle interactions, $n=$ $2,3, \ldots$ For $n$-particle interactions with $n=2,3$, where the microscopic Hamiltonian would have the form

$$
\begin{aligned}
H_{N}:=\sum_{j=1}^{N}\left(-\Delta_{x_{j}}\right) & +\frac{1}{N} \sum_{1 \leq i<j \leq N} N^{d \beta} V_{2}\left(N^{\beta}\left(x_{i}-x_{j}\right)\right) \\
& +\frac{1}{N^{2}} \sum_{1 \leq i<j<k \leq N} N^{2 d \beta} V_{3}\left(N^{\beta}\left(x_{i}-x_{j}\right), N^{\beta}\left(x_{i}-x_{k}\right)\right),
\end{aligned}
$$

a combination of the previous analysis with the one that is presented in [6] will straightforwardly produce a mean field limit described by the defocusing NLS

$$
i \partial_{t} \phi_{t}+\Delta \phi_{t}-\lambda_{2}\left|\phi_{t}\right|^{2} \phi_{t}-\lambda_{3}\left|\phi_{t}\right|^{4} \phi_{t}=0
$$

in $d=1,2$, where $\lambda_{2}=\int d x V_{2}(x) \geq 0$ and $\lambda_{3}=\int d x d x^{\prime} V_{3}\left(x, x^{\prime}\right) \geq 0$ account for the mean-field strength of the 2- and 3-body interactions.

\section{Another look at the GP hierarchy}

Proving the uniqueness of solutions to the GP hieararchy (both cubic and quintic) is the most involved part of the analysis described in Sections 2. and 3.. In order to establish uniqueness, Erdös, Schlein and Yau in [12, 13, 14] have developed very sophisticated techniques based on Feynman graph expansions. As noted in Section 2., Klainerman and Machedon have recently introduced a different method to prove the uniqueness of solutions to the GP hierarchy leading to the cubic NLS in the $d=3$ case. Their approach is based on the use of spacetime bounds on the density matrices in the GP hierarchy. However, the method introduced in [21] makes the assumption that the a priori space-time bound

$$
\left\|B_{j ; k+1} \gamma^{(k+1)}\right\|_{L_{t}^{1} \dot{H}_{k}^{1}}<C^{k}
$$

holds, with $C$ independent of $k$. The authors of [22] proved that the latter is indeed satisfied for the cubic case in $d=2$, where energy conservation in the $N$-particle system is shown to imply that $\left\|B_{j ; k+1} \gamma^{(k+1)}\right\|_{\dot{H}_{k}^{1}}<C^{k}$, even without invoking the norm in the time variable. In [6] we proved the analogous bound for the quintic case in $d=1,2$. However, it is still an open problem to prove that 
solutions to the GP hierarchy which are obtained via the limit of the BBGKY hierarchy satisfy the space-time bound in $d=3$.

Motivated by this open question, we proposed an alternative approach in [7], for proving uniqueness of solutions to both cubic and quintic GP hierarchies. The main idea of our approach is based on introducing a suitable topology on the space of sequences $\Gamma=\left(\gamma^{(k)}\right)$ of marginal density matrices using certain space-time norms. Subsequently, we establish the existence of solutions $\Gamma(t)$ to the GP hierarchy locally in time, using a fixed point argument. The uniqueness of solutions of the GP hierarchy then follows immediately. We describe this approach in more details below. Subsequently, we have continued to refine this approach in the recent papers [10], [8] and [9].

It is important to emphasize that our starting point and main focus in the papers $[7,10,8,9]$ is the GP hierarchy itself. In contrast, the starting point, for instance, of the works $[12,13,14,22,6]$, is the $N$-particle boson gas and the corresponding BBGKY hierarchy. The goal pursued in those works typically is the extraction of the NLS as a mean field limit as $N \rightarrow \infty$, while the GP hierarchy tends to be viewed as an intermediate object to reach this goal.

On the other hand, our main interest focuses on the dynamics of the GP hierarchy in general, and not specifically on factorized solutions. We note that the GP hierarchy describes the dynamics of a gas of infinitely many interacting bosons. While in general, the latter is exceedingly complicated, the Gross-Pitaevskii limit introduces some simplifying mean field features that render many aspects of it more accessible. One of our main motivations to investigate its dynamics is the question whether, and in which sense, predictions based on the mean field equations (NLS) capture the typical behavior of the manybody quantum field theory.

\subsection{The spaces}

In [7] we introduced the space

$$
\mathfrak{G}:=\bigoplus_{k=1}^{\infty} L^{2}\left(\mathbb{R}^{d k} \times \mathbb{R}^{d k}\right)
$$

of sequences of density matrices

$$
\Gamma:=\left(\gamma^{(k)}\right)_{k \in \mathbb{N}}
$$

where $\gamma^{(k)} \geq 0, \operatorname{Tr} \gamma^{(k)}=1$, and where every $\gamma^{(k)}\left(\underline{x}_{k}, \underline{x}_{k}^{\prime}\right)$ is symmetric in all components of $\underline{x}_{k}$, and in all components of $\underline{x}_{k}^{\prime}$, respectively, i.e.

$$
\gamma^{(k)}\left(x_{\pi(1)}, \ldots, x_{\pi(k)} ; x_{\pi^{\prime}(1)}^{\prime}, \ldots, x_{\pi^{\prime}(k)}^{\prime}\right)=\gamma^{(k)}\left(x_{1}, \ldots, x_{k} ; x_{1}^{\prime}, \ldots, x_{k}^{\prime}\right)
$$

holds for all $\pi, \pi^{\prime} \in S_{k}$.

The $k$-particle marginals are assumed to be hermitean,

$$
\gamma^{(k)}\left(\underline{x}_{k} ; \underline{x}_{k}^{\prime}\right)=\overline{\gamma^{(k)}\left(\underline{x}_{k}^{\prime} ; \underline{x}_{k}\right)}
$$


We call $\Gamma=\left(\gamma^{(k)}\right)_{k \in \mathbb{N}}$ admissible if $\gamma^{(k)}=\operatorname{Tr}_{k+1, \ldots, k+\frac{p}{2}} \gamma^{\left(k+\frac{p}{2}\right)}$, that is,

$$
\begin{aligned}
\gamma^{(k)} & \left(\underline{x}_{k} ; \underline{x}_{k}^{\prime}\right) \\
& =\int d x_{k+1} \cdots d x_{k+\frac{p}{2}} \gamma^{\left(k+\frac{p}{2}\right)}\left(\underline{x}_{k}, x_{k+1}, \ldots, x_{k+\frac{p}{2}} ; \underline{x}_{k}^{\prime}, x_{k+1}, \ldots, x_{k+\frac{p}{2}}\right)
\end{aligned}
$$

for all $k \in \mathbb{N}$.

Let $0<\xi<1$. Following the notation from our work [7], we define

$$
\mathcal{H}_{\xi}^{\alpha}:=\left\{\Gamma \in \mathfrak{G} \mid\|\Gamma\|_{\mathcal{H}_{\xi}^{\alpha}}<\infty\right\}
$$

where

$$
\|\Gamma\|_{\mathcal{H}_{\xi}^{\alpha}}=\sum_{k=1}^{\infty} \xi^{k}\left\|\gamma^{(k)}\right\|_{H_{k}^{\alpha}\left(\mathbb{R}^{d k} \times \mathbb{R}^{d k}\right)}
$$

with

$$
\left\|\gamma^{(k)}\right\|_{H_{k}^{\alpha}}:=\left(\operatorname{Tr}\left(\left|S^{(k, \alpha)} \gamma^{(k)}\right|^{2}\right)\right)^{\frac{1}{2}}
$$

where $S^{(k, \alpha)}:=\prod_{j=1}^{k}\left\langle\nabla_{x_{j}}\right\rangle^{\alpha}\left\langle\nabla_{x_{j}^{\prime}}\right\rangle^{\alpha}$.

The parameter $\xi>0$ is chosen in such a way that $\mathcal{H}_{\xi}^{\alpha}$ accommodates the initial data. Accordingly, $\xi$ sets the energy scale of a given Cauchy problem. If $\Gamma \in \mathcal{H}_{\xi}^{\alpha}$, then $\xi^{-1}$ is the typical $H^{\alpha}$-energy per particle.

The parameter $\alpha$ determines the regularity of the solution, and our results hold for $\alpha \in \mathfrak{A}(d, p)$ where

$$
\mathfrak{A}(d, p):=\left\{\begin{array}{cc}
\left(\frac{1}{2}, \infty\right) & \text { if } d=1 \\
\left(\frac{d}{2}-\frac{1}{2(p-1)}, \infty\right) & \text { if } d \geq 2 \text { and }(d, p) \neq(3,2) \\
{[1, \infty)} & \text { if }(d, p)=(3,2),
\end{array}\right.
$$

in dimensions $d \geq 1$, and where $p=2$ for the cubic, and $p=4$ for the quintic GP hierarchy. The parameter $\xi>0$ determines the energy scale of the problem.

Remark 1. We remark that spaces similar to $\mathcal{H}_{\xi}^{\alpha}$ are used in the isospectral renormalization group analysis of spectral problems in quantum field theory, [4].

Remark 2. We note that the norm

$$
\left\|\gamma^{(k)}\right\|_{H_{k}^{\alpha}}^{\sharp}:=\operatorname{Tr}\left(\left|S^{(k, \alpha)} \gamma^{(k)}\right|\right)
$$

is used in [12, 13]. We also employ it in [9] in connection with a priori energy bounds, together with

$$
\|\Gamma\|_{\mathcal{H}_{\xi}^{\alpha}}^{\sharp}=\sum_{k=1}^{\infty} \xi^{k}\left\|\gamma^{(k)}\right\|_{H_{k}^{\alpha}}^{\sharp}
$$

We note that

$$
\left\|\gamma^{(k)}\right\|_{H_{k}^{\alpha}} \leq C\left\|\gamma^{(k)}\right\|_{H_{k}^{\alpha}}^{\sharp}
$$

as is proven in [7]. 


\subsection{The GP hierarchy}

In [7] we introduced cubic, quintic, focusing, and defocusing GP hierarchies as follows.

Let $p \in\{2,4\}$. The $p$-GP (Gross-Pitaevskii) hierarchy is given by

$$
i \partial_{t} \gamma^{(k)}=\sum_{j=1}^{k}\left[-\Delta_{x_{j}}, \gamma^{(k)}\right]+\mu B_{k+\frac{p}{2}} \gamma^{\left(k+\frac{p}{2}\right)}
$$

in $d$ dimensions, for $k \in \mathbb{N}$. Here,

$$
B_{k+\frac{p}{2}} \gamma^{\left(k+\frac{p}{2}\right)}=B_{k+\frac{p}{2}}^{+} \gamma^{\left(k+\frac{p}{2}\right)}-B_{k+\frac{p}{2}}^{-} \gamma^{\left(k+\frac{p}{2}\right)},
$$

where

$$
B_{k+\frac{p}{2}}^{+} \gamma^{\left(k+\frac{p}{2}\right)}=\sum_{j=1}^{k} B_{j ; k+1, \ldots, k+\frac{p}{2}}^{+} \gamma^{\left(k+\frac{p}{2}\right)}
$$

and

$$
B_{k+\frac{p}{2}}^{-} \gamma^{\left(k+\frac{p}{2}\right)}=\sum_{j=1}^{k} B_{j ; k+1, \ldots, k+\frac{p}{2}}^{-} \gamma^{\left(k+\frac{p}{2}\right)}
$$

with

$$
\begin{aligned}
& \left(B_{j ; k+1, \ldots, k+\frac{p}{2}}^{+} \gamma^{\left(k+\frac{p}{2}\right)}\right)\left(t, x_{1}, \ldots, x_{k} ; x_{1}^{\prime}, \ldots, x_{k}^{\prime}\right) \\
& \quad=\int d x_{k+1} \cdots d x_{k+\frac{p}{2}} d x_{k+1}^{\prime} \cdots d x_{k+\frac{p}{2}}^{\prime} \\
& \quad \prod_{\ell=k+1}^{k+\frac{p}{2}} \delta\left(x_{j}-x_{\ell}\right) \delta\left(x_{j}-x_{\ell}^{\prime}\right) \gamma^{\left(k+\frac{p}{2}\right)}\left(t, x_{1}, \ldots, x_{k+\frac{p}{2}} ; x_{1}^{\prime}, \ldots, x_{k+\frac{p}{2}}^{\prime}\right),
\end{aligned}
$$

and

$$
\begin{aligned}
& \left(B_{j ; k+1, \ldots, k+\frac{p}{2}}^{-} \gamma^{\left(k+\frac{p}{2}\right)}\right)\left(t, x_{1}, \ldots, x_{k} ; x_{1}^{\prime}, \ldots, x_{k}^{\prime}\right) \\
& \quad=\int d x_{k+1} \cdots d x_{k+\frac{p}{2}} d x_{k+1}^{\prime} \cdots d x_{k+\frac{p}{2}}^{\prime} \\
& \quad \prod_{\ell=k+1}^{k+\frac{p}{2}} \delta\left(x_{j}^{\prime}-x_{\ell}\right) \delta\left(x_{j}^{\prime}-x_{\ell}^{\prime}\right) \gamma^{\left(k+\frac{p}{2}\right)}\left(t, x_{1}, \ldots, x_{k+\frac{p}{2}} ; x_{1}^{\prime}, \ldots, x_{k+\frac{p}{2}}^{\prime}\right) .
\end{aligned}
$$

The operator $B_{k+\frac{p}{2}} \gamma^{\left(k+\frac{p}{2}\right)}$ accounts for $\frac{p}{2}+1$-body interactions between the Bose particles. We remark that for factorized solutions

$$
\gamma^{(k)}\left(t, x_{1}, \ldots, x_{k} ; x_{1}^{\prime}, \ldots, x_{k}^{\prime}\right)=\prod_{j=1}^{k} \phi\left(t, x_{j}\right) \bar{\phi}\left(t, x_{j}^{\prime}\right),
$$


the corresponding 1-particle wave function satisfies the $p$-NLS

$$
i \partial_{t} \phi=-\Delta \phi+\mu|\phi|^{p} \phi
$$

which is focusing if $\mu=-1$, and defocusing if $\mu=+1$.

We refer to (4.11) as the cubic GP hierarchy if $p=2$, and as the quintic GP hierarchy if $p=4$. For $\mu=1$ or $\mu=-1$ we refer to the corresponding GP hierarchies as being defocusing or focusing, respectively.

The $p$-GP hierarchy can be rewritten in the following compact manner:

$$
\begin{aligned}
i \partial_{t} \Gamma+\widehat{\Delta}_{ \pm} \Gamma & =\mu \widehat{B} \Gamma \\
\Gamma(0) & =\Gamma_{0},
\end{aligned}
$$

where

$$
\widehat{\Delta}_{ \pm} \Gamma:=\left(\Delta_{ \pm}^{(k)} \gamma^{(k)}\right)_{k \in \mathbb{N}}, \quad \text { with } \Delta_{ \pm}^{(k)}=\sum_{j=1}^{k}\left(\Delta_{x_{j}}-\Delta_{x_{j}^{\prime}}\right)
$$

and

$$
\widehat{B} \Gamma:=\left(B_{k+\frac{p}{2}} \gamma^{\left(k+\frac{p}{2}\right)}\right)_{k \in \mathbb{N}}
$$

Also we use the notation

$$
\begin{aligned}
& \widehat{B}^{+} \Gamma:=\left(B_{k+\frac{p}{2}}^{+} \gamma^{\left(k+\frac{p}{2}\right)}\right)_{k \in \mathbb{N}}, \\
& \widehat{B}^{-} \Gamma:=\left(B_{k+\frac{p}{2}}^{-} \gamma^{\left(k+\frac{p}{2}\right)}\right)_{k \in \mathbb{N}} .
\end{aligned}
$$

\subsection{Local solutions to the GP hierarchy}

In our work [7] we prove local existence and uniqueness of solutions for the cubic and quintic GP hierarchy with focusing or defocusing interactions, in $\mathcal{H}_{\xi}^{\alpha}$, for $\alpha \in \mathfrak{A}(d, p)$, which satisfy a spacetime bound $\|\widehat{B} \Gamma\|_{L_{t \in I}^{1} \mathcal{H}_{\xi}^{\alpha}}<\infty$ for some $\xi>0$. This spacetime bound of Strichartz type has been conjectured by Klainerman and Machedon in [21]. We prove local existence and uniqueness of solutions which satisfy this bound via a Picard fixed point argument.

More precisely, we note that the $p$-GP hierarchy (4.13) can be formally written as a system of integral equations

$$
\begin{aligned}
\Gamma(t) & =e^{i t \widehat{\Delta}_{ \pm}} \Gamma_{0}-i \mu \int_{0}^{t} d s e^{i(t-s) \widehat{\Delta}_{ \pm}} \widehat{B} \Gamma(s) \\
\widehat{B} \Gamma(t) & =\widehat{B} e^{i t \widehat{\Delta}_{ \pm}} \Gamma_{0}-i \mu \int_{0}^{t} d s \widehat{B} e^{i(t-s) \widehat{\Delta}_{ \pm}} \widehat{B} \Gamma(s),
\end{aligned}
$$

where (4.16) is obtained by applying the operator $\widehat{B}$ on the linear non-homogeneous equation (4.15). We observe that the second line is formally a self-consistent fixed point equation for $\widehat{B} \Gamma$, which inspired us to introduce the following product space for $I:=[0, T]$ :

$$
\mathfrak{V}_{\xi}^{\alpha}(I):=L_{t \in I}^{\infty} \mathcal{H}_{\xi}^{\alpha} \times L_{t \in I}^{1} \mathcal{H}_{\xi}^{\alpha},
$$


which we endow with the norm

$$
\|(\Gamma, \Xi)\|_{\mathfrak{V}_{\xi}^{\alpha}(I)}:=\|\Gamma\|_{L_{t \in I}^{\infty} \mathcal{H}_{\xi}^{\alpha}}+\|\Xi\|_{L_{t \in I}^{1} \mathcal{H}_{\xi}^{\alpha}} .
$$

Clearly, $\left(\mathfrak{V}_{\xi}^{\alpha}(I),\|\cdot\|_{\mathfrak{V}_{\xi}^{\alpha}(I)}\right)$ is a Banach space.

Then, we introduce the system

$$
\begin{aligned}
& \Gamma(t)=e^{i t \widehat{\Delta}_{ \pm}} \Gamma_{0}-i \mu \int_{0}^{t} d s e^{i(t-s) \widehat{\Delta}_{ \pm}} \Xi(s) \\
& \Xi(t)=\widehat{B} e^{i t \widehat{\Delta}_{ \pm}} \Gamma_{0}-i \mu \int_{0}^{t} d s \widehat{B} e^{i(t-s) \widehat{\Delta}_{ \pm}} \Xi(s),
\end{aligned}
$$

which is formally equivalent to the system (4.15), (4.16).

The main local in time well-posedness result of [7] follows from the existence and uniqueness theorem that can be stated as:

Theorem 3. Assume that $\alpha \in \mathfrak{A}(d, p)$ where $d \geq 1$ and $p \in\{2,4\}$. Then, the following holds. For every $\Gamma_{0} \in \mathcal{H}_{\xi_{1}}^{\alpha}$, there exist constants $T>0$ and $0<\xi_{2} \leq \xi_{1}$ such that for $t \in I=[0, T]$, there exists a unique solution $(\Gamma(t), \Xi(t))$ of the system (4.19), (4.20), in the space $\mathfrak{V}_{\xi_{2}}^{\alpha}(I)$. Moreover, the relation

$$
\Xi(t)=\widehat{B} \Gamma(t) \in L_{t \in I}^{1} \mathcal{H}_{\xi_{2}}^{\alpha}
$$

holds for the solution, and the component $\Gamma(t)$ satisfies the p-GP hierarchy

$$
i \partial_{t} \Gamma+\widehat{\Delta}_{ \pm} \Gamma-\mu \widehat{B} \Gamma=0
$$

with initial condition $\Gamma(0)=\Gamma_{0} \in \mathcal{H}_{\xi_{1}}^{\alpha}$.

Moreover, there exists a constant $C\left(T, d, p, \xi_{1}, \xi_{2}\right)$ such that

$$
\|\widehat{B} \Gamma\|_{L_{t \in I}^{1} \mathcal{H}_{\xi_{2}}^{\alpha}} \leq C\left(T, d, p, \xi_{1}, \xi_{2}\right)\left\|\Gamma_{0}\right\|_{\mathcal{H}_{\xi_{1}}^{\alpha}}
$$

holds.

We remark that our local existence and uniqueness result differs than the one proven in $[12,13]$ in that performing the contraction principle on the space $\mathfrak{V}_{\xi_{2}}^{\alpha}(I)$ presumes finiteness of $\|\widehat{B} \Gamma\|_{L_{t \in I}^{1} \mathcal{H}_{\xi_{2}}^{\alpha}}$. However, ${ }^{\dagger}$ it is a priori conceivable that there exist solutions of the $p$-GP hierarchy $\Gamma(t) \in \mathcal{H}_{\xi_{2}}^{\alpha}$ for which $\|\widehat{B} \Gamma\|_{L_{t \in I}^{1} \mathcal{H}_{\xi_{2}}^{\alpha}}$ is not finite. In our current work in progress, we are investigating this issue further. In particular, we apply our space-time norm approach to study the limit of the BBGKY to the $p$-GP hierarchy, and to prove that all solutions to the $p$-GP hierarchy that are obtained from the BBGKY indeed satisfy this space-time bound.

The proof of Theorem 3 is based on a fixed point argument, that can be carried out thanks to:

\footnotetext{
${ }^{\dagger}$ We thank B. Schlein for calling our attention to this fact.
} 
(i) A Strichartz-type estimate for the homogeneous GP hierarchy (proved for the cubic GP when $d=3$ by Klainerman and Machedon in [21] and for the quintic GP when $d=1,2$ in our work [6]).

(ii) A combinatorial "board game argument" of [21].

Recently, in [8], we have obtained a new proof of local well-posedness for focusing and defocusing $p$-GP hierarchies, and hence of the uniqueness of solutions, based on the use of a $T-T^{*}$ type argument.

\subsection{Energy conservation and blow-up}

For defocusing cubic GP hierarchies in $d=1,2,3$, and defocusing quintic GP hierarchies in $d=1,2$, which are obtained as limits of BBGKY hierarchies as outlined in Sections 2. and 3., it is possible to derive a priori bounds on $\left\|\gamma^{(k)}(t)\right\|_{L_{t}^{\infty} H_{k}^{1}}$ based on energy conservation in the $N$-particle Schrödinger system, see [12, 13, 14], and also [6, 22]. However, on the level of the GP hierarchy, no conserved energy functional has been known. In [10], together with Tzirakis, we identify an observable corresponding to the average energy per particle, and we prove that it is conserved. More precisely, the following theorem has been proved in [10]:

Theorem 4. Let $0<\xi<1$. Assume that $\Gamma(t) \in \mathcal{H}_{\xi}^{\alpha}$, with $\alpha \geq 1$, is a solution of the focusing $(\mu=-1)$ or defocusing $(\mu=1) p$-GP hierarchy with initial condition $\Gamma_{0} \in \mathcal{H}_{\xi}^{\alpha}$. Then, the following hold. Let

$$
\begin{aligned}
E_{k}(\Gamma(t)):=\frac{1}{2} \operatorname{Tr}\left(\sum_{j=1}^{k}(\right. & \left.\left.-\Delta_{x_{j}}\right) \gamma^{(k)}(t)\right) \\
& +\frac{\mu}{p+2} \operatorname{Tr}\left(B_{k+\frac{p}{2}}^{+} \gamma^{\left(k+\frac{p}{2}\right)}(t)\right) .
\end{aligned}
$$

Then, the quantity

$$
\mathcal{E}_{\xi}(\Gamma(t)):=\sum_{k \geq 1} \xi^{k} E_{k}(\Gamma(t))
$$

is conserved, and in particular,

$$
\mathcal{E}_{\xi}(\Gamma(t))=\left(\sum_{k \geq 1} k \xi^{k}\right) E_{1}(\Gamma(t))
$$

holds, where $\sum_{k \geq 1} k \xi^{k}<\infty$ for any $0<\xi<1$.

Here, we note that $\operatorname{Tr}(A)$ refers to the integration of the kernel $A\left(x, x^{\prime}\right)$ against the measure $\int d x d x^{\prime} \delta\left(x-x^{\prime}\right)$. Moreover, we remark that for factorized states $\Gamma(t)=\left(|\phi(t)\rangle\left\langle\left.\phi(t)\right|^{\otimes k}\right)_{k \in \mathbb{N}}\right.$, one finds

$$
E_{1}(\Gamma(t))=\frac{1}{2}\|\nabla \phi(t)\|_{L^{2}}^{2}+\frac{\mu}{p+2}\|\phi(t)\|_{L^{p+2}}^{p+2}
$$


which is the usual expression for the conserved energy for solutions of the NLS $i \partial_{t} \phi+\Delta \phi+$ $\mu|\phi|^{p} \phi=0$.

Furthermore, we prove the virial identity on the level of the GP hierarchy that enables us to obtain an analogue of Glassey's argument from the analysis of focusing NLS equations. As a consequence, we prove that all solutions to the focusing GP hierarchy at the $L^{2}$-critical or $L^{2}$ supercritical level blow up in finite time if the energy per particle in the initial condition is negative.

To this end, we introduce the following observable to detect blowup of a solution of the GP hierarchy, [7].

Definition 5. We call

$$
\operatorname{Av}_{H^{\alpha}}(\Gamma):=\left[\sup \left\{\xi>0 \mid\|\Gamma\|_{\mathcal{H}_{\xi}^{\alpha}}<\infty\right\}\right]^{-1} .
$$

the average $H^{\alpha}$-energy per particle.

Clearly, $\operatorname{Av}_{H^{\alpha}}(\Gamma)$ is the reciprocal of the convergence radius of $\|\Gamma\|_{\mathcal{H}_{\xi}^{\alpha}}$, seen as a power series in $\xi$. Accordingly, the following notion of blowup of solutions is natural.

Definition 6. We say that $\Gamma(t)$ blows up in finite time with respect to $H^{\alpha}$ if there exists $0<T<\infty$ such that $\operatorname{Av}_{H^{\alpha}}(\Gamma(t)) \rightarrow \infty$ as $t \nearrow T$.

This implies that in the limit $t \nearrow T$, the solution $\Gamma(t)$ cannot be accommodated in any of the spaces $\mathcal{H}_{\xi}^{\alpha}$, for any choice of $\xi>0$. Accordingly, we establish the following negative energy blowup result.

Theorem 7. Let $p \geq p_{L^{2}}=\frac{4}{d}$ ( $L^{2}$-critical and supercritical GP hierarchy). Assume that $\Gamma(t)=$ $\left(\gamma^{(k)}(t)\right)_{k \in \mathbb{N}}$ solves the focusing (that is, $\left.\mu=-1\right) p$-GP hierarchy, with $\Gamma(0) \in \mathcal{H}_{\xi}^{1}$ for some $0<\xi<1$, and $\operatorname{Tr}\left(x^{2} \gamma^{(1)}(0)\right)<\infty$.

Then, it follows that if $E_{1}(\Gamma(0))<0$, there exists $0<T<\infty$ such that $\operatorname{Av}_{H^{1}}(\Gamma(t)) \rightarrow \infty$ as $t \nearrow T$.

For blowup solutions, we prove the following lower bounds on the blow-up rate, in [7].

Proposition 8. Assume that $\frac{4}{d} \leq p<\frac{4}{d-2 \alpha}\left(H^{1}\right.$ subcritical $)$.

If $\Gamma(t)$ blows up with respect to $H^{\alpha}$ as $t \nearrow T$, then

$$
\left(\operatorname{Av}_{H^{\alpha}}(\Gamma(t))\right)^{\frac{1}{2}}>\frac{C}{|T-t|^{\left(2 \alpha-d+\frac{4}{p}\right) / 4}}
$$

The exponent in the denominator is the same as for blowup solutions of $L^{2}$-critical and supercritical focusing NLS in the associated cases. 


\subsection{Global well-posedness for the GP hierarchy}

Recently, in [9], we have established the global well-posedness of solutions to defocusing $p$-GP hierarchies with $\Gamma_{0} \in \mathcal{H}_{\xi}^{1}$ for arbitrary $\xi>0$. This result can be understood as an improvement of the global existence and uniqueness of solutions in $\mathcal{H}_{\xi}^{1}$ which was obtained in [7] under the assumption that an a priori bound $\|\Gamma(t)\|_{\mathcal{H}_{\xi}^{1}}<c$ holds for $\xi>0$ sufficiently small. In [9], we actually prove a related a-priori bound by identifying a countably infinite family of conserved, higher order energy functionals, generalizing those found in [10], see Theorem 4. Moreover, another important tool, used in the proof of global well-posedness for defocusing $p$-GP hierarchies with $\Gamma_{0} \in \mathcal{H}_{\xi}^{1}$, is the following generalization of the Sobolev inequalities that we apply on the level of marginal density matrices.

We note that while the additive conserved energy functionals $\mathcal{E}_{\xi}(\Gamma(t))$ allow us to control the blowup of certain solutions, they are not sufficient to allow us to control the $\mathcal{H}_{\xi}^{1}$-norm, which is multiplicative with respect to the derivatives in the particle coordinates. To this end, we introduce the following family of conserved energy functionals. We define the "block operators", for $\ell \in \mathbb{N}$,

$$
K_{\ell}:=\frac{1}{2}\left(1-\Delta_{x_{\ell}}\right) \operatorname{Tr}_{\ell+1, \ldots, \ell+\frac{p}{2}}+\frac{\mu}{p+2} B_{\ell ; \ell+\frac{p}{2}}^{+}
$$

$K_{\ell}$ affects only block of $k_{p}:=1+\frac{p}{2}$ variables, starting at index $\ell$, in the density matrix

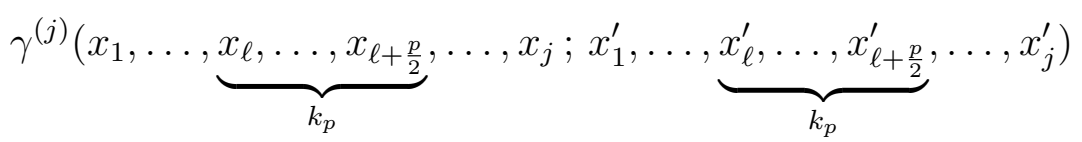

for $j \geq \ell+k_{p}$.

The operator $K_{\ell}$ is related to the one-particle energy $E_{1}(\Gamma)$ in the following way, owing to admissibility.

$$
\operatorname{Tr}_{1, \ldots, \ell, \ell+k_{p}, \cdots, j}\left[K_{\ell} \gamma^{(j)}\right]=\frac{1}{2}+E_{1}(\Gamma)
$$

Next, we assemble $m$ successive blocks into the operator

$$
\mathcal{K}^{(m)}:=K_{1} K_{k_{p}+1} \cdots K_{(m-1) k_{p}+1} .
$$

We then prove the following conservation law for the GP hierarchy.

Theorem 9. Assume that $\Gamma=\left(\gamma^{(j)}\right)$ is an admissible solution of the p-GP hierarchy, for focusing or defocusing interactions, $\mu= \pm 1$. Then,

$$
\left\langle\mathcal{K}^{(m)}\right\rangle_{\Gamma(t)}:=\operatorname{Tr}_{1, k_{p}+1,2 k_{p}+1, \ldots,(m-1) k_{p}+1}\left[\mathcal{K}^{(m)} \gamma^{\left(m k_{p}\right)}(t)\right]
$$

is conserved. Likewise, the higher order conserved $\xi$-energy

$$
\mathcal{K}_{\xi}(\Gamma(t)):=\sum_{k=1}^{\infty}(2 \xi)^{k}\left\langle\mathcal{K}^{(m)}\right\rangle_{\Gamma(t)}=\mathcal{K}_{\xi}(\Gamma(0))
$$

is conserved. 
Using the conservation of this higher order energy, we prove well-posedness of solutions of energy subcritical, defocusing $p$-GP hierarchies.

Theorem 10. Assume $p<\frac{4}{d-2}$ and $1 \in \mathfrak{A}(d, p)$, and $\left\|\Gamma_{0}\right\|_{\mathcal{H}_{\xi^{\prime}}^{1}}^{\sharp}<\infty$ for $0<\xi^{\prime}<1$. Let $C_{\text {Sob }}$ be the constant for the generalized Sobolev inequality in Proposition (11) below, and

$$
\xi \leq\left(1+\frac{2}{p+2} C_{S o b}\right)^{-\frac{1}{k_{p}}} \xi^{\prime}
$$

Let $\mathbb{R}=\cup_{j} I_{j}$ with $I_{j}:=[j T,(j+1) T]$, where $T<T_{0}(d, p, \xi)$ denotes the local well-posedness time from Theorem 3. Then, there exists a unique global solution $\Gamma \in \cup_{j \in \mathbb{Z}} \mathcal{W}_{\xi}^{1}\left(I_{j}\right)$, where

$$
\mathcal{W}_{\xi}^{\alpha}(I)=\left\{\Gamma \in L_{t \in I}^{\infty} \mathcal{H}_{\xi}^{\alpha} \mid \widehat{B} \Gamma \in L_{t \in I}^{2} \mathcal{H}_{\xi}^{\alpha}\right\}
$$

of the defocusing $p$-GP hierarchy with

$$
\|\Gamma(t)\|_{\mathcal{H}_{\xi}^{1}} \leq\|\Gamma(t)\|_{\mathcal{H}_{\xi}^{1}}^{\sharp} \leq \sum_{m \in \mathbb{N}}(2 \xi)^{m}\left\langle\mathcal{K}^{(m)}\right\rangle_{\Gamma_{0}} \leq\left\|\Gamma_{0}\right\|_{\mathcal{H}_{\xi^{\prime}}^{1}}^{\sharp} \quad \forall t \in \mathbb{R} .
$$

The first inequality in (4.28) is always true. The second inequality follows from the positivity of the interaction energy per particle in the defocusing case. The last inequality is obtained from bounding the interaction energy of each particle with the mean field by the following generalized Sobolev inequality.

Proposition 11. (Generalized Sobolev inequality.) Assume that $f \in H^{\alpha}\left(\mathbb{R}^{q d}\right)$. Then, there exists a constant $0<C_{S o b}<\infty$ such that

$$
\begin{aligned}
& \left(\int d x|f(\underbrace{x, \ldots, x}_{q})|^{2}\right)^{\frac{1}{2}} \\
& \leq C_{S o b}\left(\int d x_{1} \cdots d x_{q}\left|\left\langle\nabla_{x_{1}}\right\rangle^{\alpha} \cdots\left\langle\nabla_{x_{q}}\right\rangle^{\alpha} f\left(x_{1}, \ldots, x_{q}\right)\right|^{2}\right)^{\frac{1}{2}} \\
& =C_{S o b}\|f\|_{H_{x_{1}, \ldots, x_{q}}^{\alpha}}
\end{aligned}
$$

for $\alpha>\frac{(q-1) d}{2 q}$ and $x_{i} \in \mathbb{R}^{d}$.

The left hand side,

$$
f(x, \ldots, x)=\int d \xi_{1} \cdots d \xi_{q} \widehat{f}\left(\xi_{1}, \ldots, \xi_{q}\right) e^{i x\left(\xi_{1}+\cdots+\xi_{q}\right)},
$$

is defined via the $L^{2}$-Fourier transform of $f \in H^{\alpha}\left(\mathbb{R}^{q d}\right)$.

Moreover, we prove the global well-posedness of solutions for focusing and defocusing $p$-GP hierarchies on the $L^{2}$-subcritical level, $p<\frac{4}{d}$, [8]. 
Theorem 12. Assume $p<p_{L^{2}}=\frac{4}{d}$ ( $L^{2}$-subcritical level). Moreover, let $\Gamma_{0} \in \mathcal{H}_{\xi^{\prime}}^{1 \sharp}$ for some $0<\xi^{\prime}<1$, and $1 \in \mathfrak{A}(d, p)$. Then, for $0<\xi<1$ sufficiently small (depending on $\xi^{\prime}$ ), the following holds:

There exists a unique global solution $\Gamma \in \cup_{j \in \mathbb{Z}} \mathcal{W}_{\xi}^{1}\left(I_{j}\right)$ of the focusing or defocusing $p-G P$ hierarchy such that, $\forall t \in \mathbb{R}$,

$$
\|\Gamma(t)\|_{\mathcal{H}_{\xi}^{1}} \leq\|\Gamma(t)\|_{\mathcal{H}_{\xi}^{1}}^{\sharp} \leq 2 \sum_{m \in \mathbb{N}}(2 \xi)^{m}\left\langle\mathcal{K}^{(m)}\right\rangle_{\Gamma_{0}} \leq C\left(d, p, \xi / \xi^{\prime}\right)\left\|\Gamma_{0}\right\|_{\mathcal{H}_{\xi^{\prime}}^{1}}^{\sharp}
$$

This result again relies on the the existence of the higher order conserved energy functionals, Theorem 9. The last inequality in (4.29) is proven by recursively applying the following generalized Gagliardo-Nirenberg inequalities, which we use to dominate the interaction term by the kinetic term, for both focusing and defocusing GP hierarchies.

Proposition 13. (Generalized Gagliardo-Nirenberg inequality.) Assume that $f \in H^{1}\left(\mathbb{R}^{q d}\right)$ and $1 \geq \alpha>\frac{(q-1) d}{2 q}$. Then, there exists a constant $0<C_{G N}<\infty$ such that

$$
\left(\int d x|f(\underbrace{x, \ldots, x}_{q})|^{2}\right)^{\frac{1}{2}} \leq C_{G N}\|f\|_{H_{x_{1}, \ldots, x_{q}}^{1}}^{\alpha}\|f\|_{L_{x_{1}, \ldots, x_{q}}^{2}}^{1-\alpha}
$$

where $x_{i} \in \mathbb{R}^{d}$.

This result is a corollary of Proposition 11.

\section{Acknowledgements}

In the course of our works presented in this review article, we have benefitted very much from very useful comments due to J. Colliander, D. Hundertmark, S. Klainerman, M. Lewin, I. Rodnianski, N. Tzirakis, M. Weinstein, and especially from B. Schlein and H.-T. Yau. The work of T.C. was supported by NSF grant DMS 0704031 / DMS-0940145. The work of N.P. was supported NSF grant number DMS 0758247 and an Alfred P. Sloan Research Fellowship.

\section{References}

[1] R. Adami, G. Golse, A. Teta. Rigorous derivation of the cubic NLS in dimension one. J. Stat. Phys., 127 (2007), No. 6, 1194-1220.

[2] M. Aizenman, E.H. Lieb, R. Seiringer, J.P. Solovej, J. Yngvason. Bose-Einstein Quantum Phase Transition in an Optical Lattice Model. Phys. Rev. A, 70 (2004), 023612.

[3] I. Anapolitanos, I.M. Sigal. The Hartree-von Neumann limit of many body dynamics. Preprint http://arxiv.org/abs/0904.4514. 
[4] V. Bach, T. Chen, J. Fröhlich and I.M. Sigal. Smooth Feshbach map and operator-theoretic renormalization group methods. J. Funct. Anal., 203 (2003), No. 1, 44-92.

[5] T. Cazenave. Semilinear Schrödinger equations. Courant lecture notes, 10 (2003), Amer. Math. Soc..

[6] T. Chen, N. Pavlović. The quintic NLS as the mean field limit of a Boson gas with three-body interactions. J. Functional Analysis, conditionally accepted. Preprint http://arxiv.org/abs/0812.2740.

[7] T. Chen, N. Pavlović. On the Cauchy problem for focusing and defocusing Gross-Pitaevskii hierarchies. Discr. Contin. Dyn. Syst., 27 (2010), No. 2, 715 - 739.

[8] T. Chen, N. Pavlović. A short proof of local wellposedness for focusing and defocusing Gross-Pitaevskii hierarchies. Preprint http://arxiv.org/abs/0906.3277.

[9] T. Chen, N. Pavlović. Higher order energy conservation, Gagliardo-NirenbergSobolev inequalities, and global well-posedness for Gross-Pitaevskii hierarchies. Preprint http://arxiv.org/abs/0906.2984.

[10] T. Chen, N. Pavlović, N. Tzirakis. Energy conservation and blowup of solutions for focusing GP hierarchies. Preprint http://arXiv.org/abs/0905.2704.

[11] A. Elgart, L. Erdös, B. Schlein, H.-T. Yau. Gross-Pitaevskii equation as the mean field limit of weakly coupled bosons. Arch. Rat. Mech. Anal., 179 (2006), No. 2, 265-283.

[12] L. Erdös, B. Schlein, H.-T. Yau. Derivation of the Gross-Pitaevskii hierarchy for the dynamics of Bose-Einstein condensate. Comm. Pure Appl. Math., 59 (2006), No. 12, 1659-1741.

[13] L. Erdös, B. Schlein, H.-T. Yau. Derivation of the cubic non-linear Schrödinger equation from quantum dynamics of many-body systems. Invent. Math., 167 (2007), 515-614.

[14] L. Erdös, H.-T. Yau. Derivation of the nonlinear Schrödinger equation from a many body Coulomb system. Adv. Theor. Math. Phys., 5 (2001), No. 6, 1169-1205.

[15] J. Fröhlich, S. Graffi, S. Schwarz. Mean-field- and classical limit of many-body Schrödinger dynamics for bosons. Comm. Math. Phys., 271 (2007), no. 3, 681-697.

[16] J. Fröhlich, A. Knowles, A. Pizzo. Atomism and quantization. J. Phys. A, 40 (2007), No. 12, 3033-3045.

[17] J. Fröhlich, A. Knowles, S. Schwarz. On the Mean-Field Limit of Bosons with Coulomb Two-Body Interaction. Preprint arXiv:0805.4299.

[18] M. Grillakis, M. Machedon, A. Margetis. Second-order corrections to mean field evolution for weakly interacting Bosons I. Preprint http://arxiv.org/abs/0904.0158. 
[19] M. Grillakis, A. Margetis. A priori estimates for many-body Hamiltonian evolution of interacting boson system. J. Hyperbolic Differ. Equ., 5 (2008), No. 4, 857-883.

[20] K. Hepp. The classical limit for quantum mechanical correlation functions. Comm. Math. Phys., 35 (1974), 265-277.

[21] S. Klainerman, M. Machedon. On the uniqueness of solutions to the Gross-Pitaevskii hierarchy. Commun. Math. Phys., 279 (2008), No. 1, 169-185.

[22] K. Kirkpatrick, B. Schlein, G. Staffilani. Derivation of the two dimensional nonlinear Schrödinger equation from many body quantum dynamics. Preprint arXiv:0808.0505.

[23] E.H. Lieb, R. Seiringer. Proof of Bose-Einstein condensation for dilute trapped gases. Phys. Rev. Lett., 88 (2002), 170409.

[24] E.H. Lieb, R. Seiringer, J.P. Solovej, J. Yngvason. The mathematics of the Bose gas and its condensation. Birkhäuser (2005).

[25] E.H. Lieb, R. Seiringer, J. Yngvason. A rigorous derivation of the Gross-Pitaevskii energy functional for a two-dimensional Bose gas. Commun. Math. Phys., 224 (2001) No. 1, 17-31.

[26] I. Rodnianski, B. Schlein. Quantum fluctuations and rate of convergence towards mean field dynamics. Comm. Math. Phys., 29 (2009), No. 1, 31-611.

[27] B. Schlein. Derivation of Effective Evolution Equations from Microscopic Quantum Dynamics. Lecture notes for the minicourse held at the 2008 CMI Summer School in Zurich.

[28] H. Spohn. Kinetic Equations from Hamiltonian Dynamics. Rev. Mod. Phys. 52 (1980), No. 3, 569-615.

[29] T. Tao. Nonlinear dispersive equations. Local and global analysis. CBMS 106 (2006), AMS. 